\title{
Competing electronic instabilities in the quadruple perovskite manganite $\mathrm{PbMn7012}$
}

\author{
R. D. Johnson, D. D. Khalyavin, P. Manuel, and A. A. Belik
}

\section{Published version information}

Citation: RD Johnson et al. Competing electronic instabilities in the quadruple perovskite manganite PbMn7012. Phys Rev B 103, no. 13 (2021): 134115

DOI: $10.1103 /$ PhysRevB.103.134115

This version is made available in accordance with publisher policies. Please cite only the published version using the reference above. This is the citation assigned by the publisher at the time of issuing the APV. Please check the publisher's website for any updates. 


\title{
Competing electronic instabilities in the quadruple perovskite manganite $\mathrm{PbMn}_{7} \mathrm{O}_{12}$
}

\author{
R. D. Johnson, ${ }^{1, *}$ D. D. Khalyavin, ${ }^{2}$ P. Manuel, ${ }^{2}$ and A. A. Belik $\oplus^{3}$ \\ ${ }^{1}$ Department of Physics and Astronomy, University College London, Gower Street, London, WC1E 6BT, United Kingdom \\ ${ }^{2}$ ISIS facility, Rutherford Appleton Laboratory-STFC, Chilton, Didcot, OX11 0QX, United Kingdom \\ ${ }^{3}$ International Center for Materials Nanoarchitectonics (WPI-MANA), National Institute for Materials Science (NIMS), \\ Namiki 1-1, Tsukuba, Ibaraki 305-0044, Japan
}

(Received 23 February 2021; accepted 12 April 2021; published 21 April 2021)

\begin{abstract}
Structural behavior of $\mathrm{PbMn}_{7} \mathrm{O}_{12}$ has been studied by high resolution synchrotron x-ray powder diffraction. This material belongs to a family of quadruple perovskite manganites that exhibit an incommensurate structural modulation associated with an orbital density wave. It has been found that the structural modulation in $\mathrm{PbMn}_{7} \mathrm{O}_{12}$ onsets at $294 \mathrm{~K}$ with the incommensurate propagation vector $\mathbf{k}_{s}=(0,0, \sim 2.08)$. At $110 \mathrm{~K}$ another structural transition takes place where the propagation vector suddenly drops down to a quasicommensurate value $\mathbf{k}_{s}=(0,0,2.0060(6))$. The quasicommensurate phase is stable in the temperature range of 40-110 K, and below $40 \mathrm{~K}$ the propagation vector jumps back to the incommensurate value $\mathbf{k}_{s}=(0,0, \sim 2.06)$. Both low temperature structural transitions are strongly first order with large thermal hysteresis. The orbital density wave in the quasicommensurate phase has been found to be substantially suppressed in comparison with the incommensurate phases, which naturally explains unusual magnetic behavior recently reported for this perovskite. Analysis of the refined structural parameters revealed that that the presence of the quasicommensurate phase is likely to be associated with a competition between the $\mathrm{Pb}^{2+}$ lone electron pair and $\mathrm{Mn}^{3+} \mathrm{Jahn}-$ Teller instabilities.
\end{abstract}

DOI: 10.1103/PhysRevB.103.134115

\section{INTRODUCTION}

The Jahn-Teller (JT) effect in transition metal compounds presents a direct interaction between the electronic configuration of the transition metal ions and the crystal structure. As such, it can mediate long-range ordering and cross coupling of orbital, charge, and magnetic degrees of freedom [1,2] as exemplified by the simple perovskite manganites with generic chemical formula $A_{1-x} A_{x}^{\prime} B \mathrm{O}_{3}(B=\mathrm{Mn})$ [3]. For example, in $\mathrm{La}_{0.5} \mathrm{Ca}_{0.5} \mathrm{MnO}_{3} \mathrm{JT}$-active $\mathrm{Mn}^{3+}$ and non-JT-active $\mathrm{Mn}^{4+}$ ions adopt a 1:1 checkerboard type charge order. All $\mathrm{Mn}^{3+}$ octahedral oxygen coordinations coherently elongate according to their JT instability, giving rise to long-range ordering of the $\mathrm{Mn}^{3+} d_{3 z^{2}-r^{2}}$ orbitals. These orbitals in turn mediate ferromagnetic superexchange interactions within zigzag chains, which then couple antiferromagnetically to form a so-called CE-type magnetic structure $[4,5]$.

A more complex pattern of magneto-orbital order preceded by charge order has been found in the quadruple perovskite manganites with generic chemical formula $A A_{3}^{\prime} B_{4} \mathrm{O}_{12}\left(A^{\prime}=\right.$ $B=\mathrm{Mn}$ ). For example, in $\mathrm{CaMn}_{7} \mathrm{O}_{12}$ 3:1 charge ordering of $B$ site $\mathrm{Mn}^{3+}$ and $\mathrm{Mn}^{4+}$ ions leads to a trigonal structural distortion that compresses the JT-active $\mathrm{Mn}^{3+}$ octahedra, leading to an apparent ordering of $d_{x^{2}-y^{2}}$ orbitals [6]. However, anharmonic contributions to the elastic energy favor elongated rather than compressed $\mathrm{Mn}^{3+}$ octahedra [7], which results in an incommensurate orbital modulation (orbital density wave) that propagates along the trigonal axis with wave vector $\mathbf{k}_{s}=$

*roger.johnson@ucl.ac.uk $\left(0,0, k_{z}\right)$. The orbital density wave is characterized by an oscillation in the orbital mixing between $d_{3 x^{2}-r^{2}}$ and $d_{3 y^{2}-r^{2}}$, via $d_{x^{2}-y^{2}}$ [8]. Using the superspace formalism, this modulated state is described by the $R \overline{3}(00 \gamma) 0$ symmetry [8,9]. At lower temperatures the incommensurate orbital modulation is accompanied by an incommensurate magnetic structure with a fundamental propagation vector $\mathbf{k}_{0}$ that initially locks into the periodicity of the orbital modulation with $\mathbf{k}_{0}=\mathbf{k}_{s} / 2$, and then delocks at a second magnetic phase transition below which the system establishes its ground state [10-12]. In the delocked ground state the magnetic structure is a constant moment helix with an incommensurately modulated spin chirality evidenced by higher order magnetic diffraction peaks with propagation vectors $\mathbf{k}_{n+}=n \mathbf{k}_{s}+\mathbf{k}_{0}$ and $\mathbf{k}_{n-}=$ $n \mathbf{k}_{s}-\mathbf{k}_{0}$, where $n=1,2,3 \ldots, \infty$ [13]. This type of magnetic structure is stabilized by a set of competing exchange interactions and magnetic anisotropies modulated by the orbital density wave-an effect referred to as magneto-orbital coupling.

The substitution of trivalent $\mathrm{Bi}$ for divalent $\mathrm{Ca}$ in $\mathrm{BiMn}_{7} \mathrm{O}_{12}$ makes all $B$ site $\mathrm{Mn}$ ions adopt a +3 oxidation state. Hence, the 3:1 charge ordering and the incommensurate orbital helix are eliminated and replaced by a commensurate JT-driven orbital order $[14,15]$ analogous to that found in the simple perovskite $\mathrm{LaMnO}_{3}$ [16]. However, $\mathrm{BiMn}_{7} \mathrm{O}_{12}$ supports a second, polar structural instability associated with the $\mathrm{Bi}^{3+}$ lone-pair electrons, which gives rise to two, low symmetry ferroelectric phases not found in the divalent $A$ site quadruple perovskites $[14,15,17]$. Remarkably, an incommensurate orbital modulation can be established through light hole doping in $\mathrm{BiCu}_{0.1} \mathrm{Mn}_{6.9} \mathrm{O}_{12}$ [18]. In this doped material 
the incommensurate orbital order competes with the polar instabilities of the $\mathrm{Bi}^{3+}$ lone-pair electrons, which are forced to abandon their commensurate ferroelectric order and instead form the first example of a spontaneous incommensurate electric dipole helix [18].

In this paper we have brought together concepts established for $\mathrm{CaMn}_{7} \mathrm{O}_{12}$ and $\mathrm{BiMn}_{7} \mathrm{O}_{12}$, and applied them to $\mathrm{PbMn}_{7} \mathrm{O}_{12}$ - a divalent $A$ site quadruple perovskite in which one might expect the competition between lone-pair and JT instabilities to play an important role in orbital and magnetic ordering. Indeed, the magnetic behavior of this perovskite has been found to be substantially different from other members of the $A^{2+} \mathrm{Mn}_{7} \mathrm{O}_{12}$ family [19]. Apart from the highertemperature lock-in phase and the delocked ground state with modulated spin chirality, $\mathrm{PbMn}_{7} \mathrm{O}_{12}$ exhibits an additional intermediate magnetic phase in which the magnetic propagation vector is delocked from the structural modulation, but no modulation of the spin chirality was detected [19]. As such, this intermediate phase appears to contradict the phenomenological model developed to explain magneto-orbital coupling in the $A^{2+} \mathrm{Mn}_{7} \mathrm{O}_{12}$ manganites [13].

We present a detailed crystallographic study of the low-temperature phases of $\mathrm{PbMn}_{7} \mathrm{O}_{12}$ in which we have discovered a new structural phase that spans both the lock-in magnetic phase and the intermediate magnetic phase. We demonstrate that this structural phase is characterized by an approximately commensurate structural modulation $\left[\mathbf{k}_{s}=\right.$ $(0,0,2.0060(6))]$ that satisfies the lone-pair instability of $2 / 3 \mathrm{~Pb}^{3+}$ ions, but at considerable cost to the orbital order. These results reconcile the apparent discrepancy between the reported intermediate magnetic phase and the expected phenomenology. Furthermore, they present a scenario in which the competition between lone-pair and JT instabilities is intimately linked to active charge degrees of freedom that may be tunable by appropriate doping schemes. Hence, $\mathrm{PbMn}_{7} \mathrm{O}_{12}$ brings fresh paradigms to the rich magneto-orbital physics of the divalent $A$ site quadruple perovskite manganites.

\section{EXPERIMENT}

A polycrystalline sample of $\mathrm{PbMn}_{7} \mathrm{O}_{12}$ was prepared from stoichiometric mixtures of $\mathrm{Mn}_{2} \mathrm{O}_{3}$ (99.997\%), $\mathrm{MnO}_{1.839}$ (Alpha Aesar $\mathrm{MnO}_{2} 99.997 \%$ with the precise oxygen content determined by thermogravimetric analysis), and $\mathrm{PbO}$ (99.999\%). The mixtures were placed in Au capsules and treated at $6 \mathrm{GPa}$ and $1373 \mathrm{~K}$ for $2 \mathrm{~h}$ (the duration of heating to the desired temperatures was $10 \mathrm{~min}$ ) in a belt-type highpressure apparatus. After the heat treatments, the samples were quenched to room temperature, and the pressure was slowly released. Synchrotron x-ray powder diffraction data were collected using the CRISTAL beamline at SOLEIL, the French national synchrotron facility. A fine polycrystalline sample of $\mathrm{PbMn}_{7} \mathrm{O}_{12}$ was loaded into a $0.3 \mathrm{~mm}$ diameter Lindemann capillary. The capillary was then mounted within a ${ }^{4} \mathrm{He}$ flow cryostat installed on a 2 -circle, high resolution powder diffractometer. Diffraction patterns were collected on cooling from $290 \mathrm{~K}$ down to a base temperature of $5 \mathrm{~K}$, and on warming from 5 up to $300 \mathrm{~K}$. An x-ray wavelength of $0.58147 \AA$ was selected for all measurements. Structural refinements were performed using JANA2006 [20].
TABLE I. $R \overline{3}$ crystal structure parameters of $\mathrm{PbMn}_{7} \mathrm{O}_{12}$ refined at $300,160,100$, and $20 \mathrm{~K}$. Below $T_{\mathrm{OO} 1}$, these parameters correspond to the average, unmodulated structure. Atomic Wyckoff positions are as follows. Pb: $3 a[0,0,0], \mathrm{Mn} 1: 9 e\left[\frac{1}{2}, 0,0\right], \mathrm{Mn} 2: 9 d\left[\frac{1}{2}, 0, \frac{1}{2}\right]$, $\mathrm{Mn} 3: 3 b\left[0,0, \frac{1}{2}\right], \mathrm{O} 1$ and $\mathrm{O} 2: 18 f[x, y, z] . U_{\text {iso }}$ is given in units of $\AA^{2}$.

\begin{tabular}{|c|c|c|c|c|}
\hline$T(\mathrm{~K})$ & 300 & 160 & 100 & 20 \\
\hline \multicolumn{5}{|c|}{ Lattice parameters } \\
\hline$a(\AA)$ & $10.5251(3)$ & $10.5138(2)$ & $10.5099(2)$ & $10.5087(2)$ \\
\hline$c(\AA)$ & $6.4121(1)$ & $6.4109(1)$ & $6.4115(2)$ & $6.4094(1)$ \\
\hline \multicolumn{5}{|c|}{ Fractional coordinates and a.d.p.s } \\
\hline $\mathrm{Pb} \quad U_{\text {iso }}$ & $0.00651(7)$ & $0.00651(6)$ & $0.00521(6)$ & $0.00405(5)$ \\
\hline Mn1 $U_{\text {iso }}$ & $0.0065(1)$ & $0.0056(2)$ & $0.0057(2)$ & $0.0042(2)$ \\
\hline $\operatorname{Mn} 2 U_{\text {iso }}$ & $0.0027(1)$ & $0.0041(1)$ & $0.0034(2)$ & $0.0033(1)$ \\
\hline $\operatorname{Mn} 3 U_{\text {iso }}$ & $0.0030(3)$ & $0.0048(3)$ & $0.0044(3)$ & $0.0042(3)$ \\
\hline \multirow[t]{4}{*}{$\mathrm{O} 1$} & $0.2269(4)$ & $0.2274(3)$ & $0.2271(3)$ & $0.2272(3)$ \\
\hline & $0.2815(4)$ & $0.2792(3)$ & $0.2784(4)$ & $0.2786(3)$ \\
\hline & $0.0796(4)$ & $0.0819(4)$ & $0.0822(4)$ & $0.0817(4)$ \\
\hline & $0.0052(7)$ & $0.0041(7)$ & $0.0048(7)$ & $0.0037(6)$ \\
\hline \multirow[t]{4}{*}{$\mathrm{O} 2$} & $0.3433(3)$ & $0.3417(3)$ & $0.3418(3)$ & $0.3419(3)$ \\
\hline & $0.5217(3)$ & $0.5208(3)$ & $0.5212(3)$ & $0.5207(3)$ \\
\hline & $0.3346(5)$ & $0.3339(4)$ & $0.3350(5)$ & $0.3347(4)$ \\
\hline & $0.0011(6)$ & $0.0037(8)$ & $0.0045(8)$ & $0.0035(7)$ \\
\hline \multicolumn{5}{|c|}{ Reliability parameters (main reflections) } \\
\hline$R(\%)$ & 2.5 & 1.1 & 1.1 & 0.9 \\
\hline$w R(\%)$ & 3.0 & 1.4 & 1.4 & 1.2 \\
\hline
\end{tabular}

\section{RESULTS}

At high temperature $\mathrm{PbMn}_{7} \mathrm{O}_{12}$ adopts a cubic crystal structure (space group $\operatorname{Im} \overline{3}$ ) common to other divalent $A$ site quadruple perovskites [21]. In this structure, $\mathrm{Pb}^{2+}$ ions are 12-fold coordinated at the $A$ site, $\mathrm{Mn}^{3+}$ ions are located in square-planar coordinations at the $A^{\prime}$ site (labeled Mn1), and all $B$ sites are symmetry equivalent and occupied by Mn with an average oxidation state of +3.25 . A structural phase transition associated with a $3: 1 \mathrm{~B}$ site charge ordering of $\mathrm{Mn}^{3+}$ (labeled $\mathrm{Mn} 2$ ) and $\mathrm{Mn}^{4+}$ (labeled $\mathrm{Mn} 3$ ), respectively, was reported to occur in the temperature range 380-397 $\mathrm{K}$ [21,22]. The charge ordering is accompanied by a trigonal distortion of the crystal structure (space group $R \overline{3}$ ) and compression of the JT active $B$ site $\mathrm{Mn}^{3+}$ octahedra [21,22]. This $R \overline{3}$ crystal structure was refined against our synchrotron powder diffraction data measured at $300 \mathrm{~K}$ and was found to be in excellent agreement. The refined structural parameters are given in Table I. Two trace impurity phases were identified as $\alpha-\mathrm{Mn}_{2} \mathrm{O}_{3}\left(2.0\right.$ wt. \%) and $\mathrm{Pb}_{3}\left(\mathrm{CO}_{3}\right)_{2}(\mathrm{OH})_{2}(0.7$ wt. \%).

A second structural phase transition at $T_{\mathrm{OO} 1}=294 \mathrm{~K}$ was also reported, and was assigned to the onset of orbital order [22]. Below $T \sim T_{\mathrm{OO} 1}$, a large number of weak peaks appeared in our diffraction data. These peaks could be indexed as satellite reflections of the main structural Bragg peaks, reached by the incommensurate propagation vector $\mathbf{k}_{s}=$ $\left(0,0, k_{z}\right)$. The square root of the superimposed $(5, \overline{6}, 2)-\mathbf{k}_{\mathrm{s}}$ and $(6, \overline{5}, 2)-\mathbf{k}_{\mathrm{s}}$ integrated satellite intensities [marked by an asterisk in the inset to Fig. 2(a)] is shown in Fig. 1(a), which demonstrates that an incommensurate structural modulation persisted from $T_{\mathrm{OO} 1}$ down to $5 \mathrm{~K}$. 


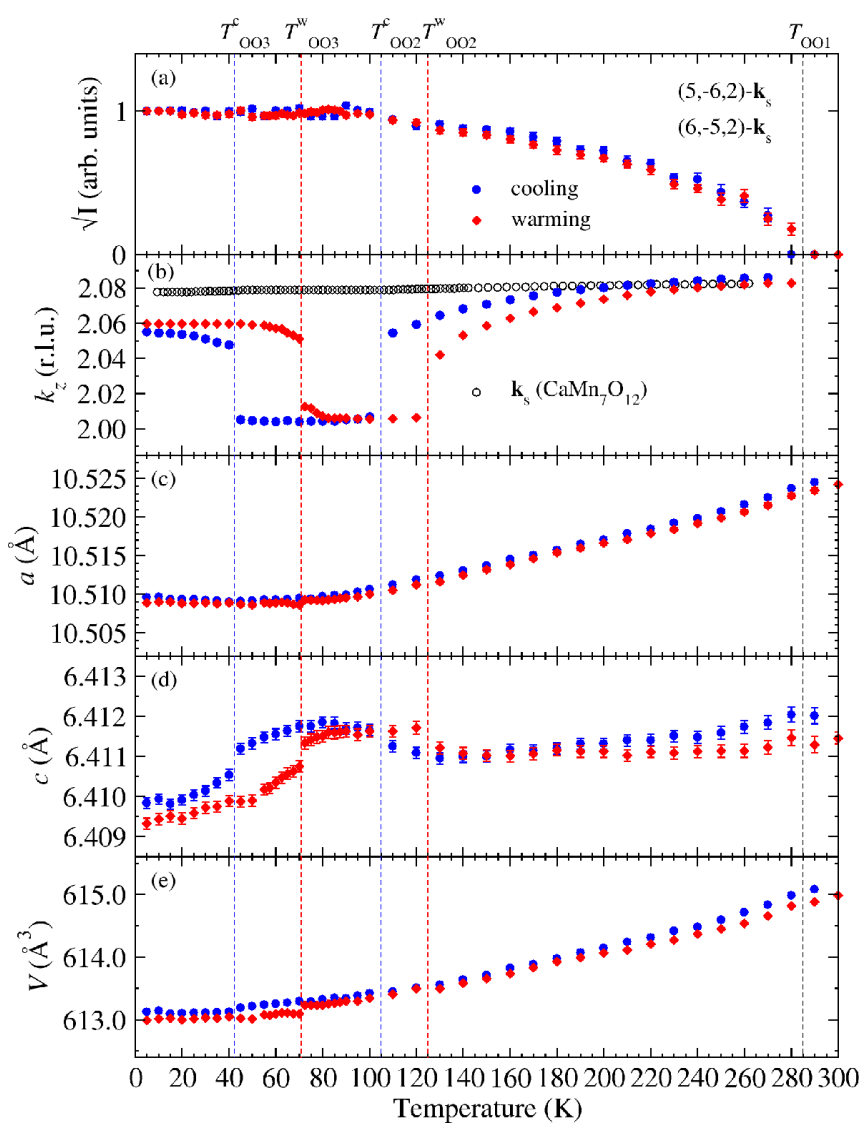

FIG. 1. Temperature dependence of (a) the square root of the superimposed $(5, \overline{6}, 2)-\mathbf{k}_{\mathrm{s}}$ and $(6, \overline{5}, 2)-\mathbf{k}_{\mathrm{s}}$ integrated satellite intensities, (b) the $z$ component of $\mathbf{k}_{\mathrm{s}}$, (c) the $a$ lattice parameter, (d) the $c$ lattice parameter, and (e) the unit cell volume. Values refined on cooling and warming are shown as blue circles and red diamonds, respectively.

The temperature dependence of $k_{z}$ is shown in Fig. 1(b), alongside the temperature dependence of the same propagation vector component determined for $\mathrm{CaMn}_{7} \mathrm{O}_{12}$ (reproduced from Ref. [13]). Immediately below $T_{\mathrm{OO} 1}$ both $\mathrm{PbMn}_{7} \mathrm{O}_{12}$ and $\mathrm{CaMn}_{7} \mathrm{O}_{12}$ supported structural modulations with $\mathbf{k}_{s}=$ $(0,0, \sim 2.08)$. However, on cooling $\mathrm{PbMn}_{7} \mathrm{O}_{12}$ below $T_{\mathrm{OO} 2}^{\mathrm{c}}=$ $105 \mathrm{~K}$ (superscript denotes cooling) there occurred a striking departure from the structural behavior of $\mathrm{CaMn}_{7} \mathrm{O}_{12}$, marked by a downward step in $k_{z}$ to the quasicommensurate position $\mathbf{k}_{s}=(0,0,2.0060(6))$ [Fig. 1(b)]. We note that the resolution of our diffraction experiment was sufficient to observe a significant difference from the commensurate vector $(0,0,2)$. The step in $k_{z}$ at $T_{\mathrm{OO} 2}^{\mathrm{c}}$ was accompanied by an anomalous increase in the $c$ lattice parameter [Fig. 1(d)], indicating a significant strain coupling between the structural modulation and the lattice. This quasicommensurate structural phase was found to persist down to $T_{\mathrm{OO} 3}^{\mathrm{c}}=43 \mathrm{~K}$, at which point $k_{z}$ and $c$ stepped back towards values measured above $T_{\mathrm{OO} 2}$. On warming, a large thermal hysteresis of width 28 and $20 \mathrm{~K}$ was observed at $T_{\mathrm{OO} 3}$ and $T_{\mathrm{OO} 2}$, respectively (Fig. 1).

Microscopic details of the structural modulation in all three incommensurate phases $\left(T<T_{\mathrm{OO} 3}, T_{\mathrm{OO} 3}<T<T_{\mathrm{OO} 2}\right.$, and $T_{\mathrm{OO} 2}<T<T_{\mathrm{OO} 1}$ ) were established through refinement of a structural model defined within a 4D superspace formalism.

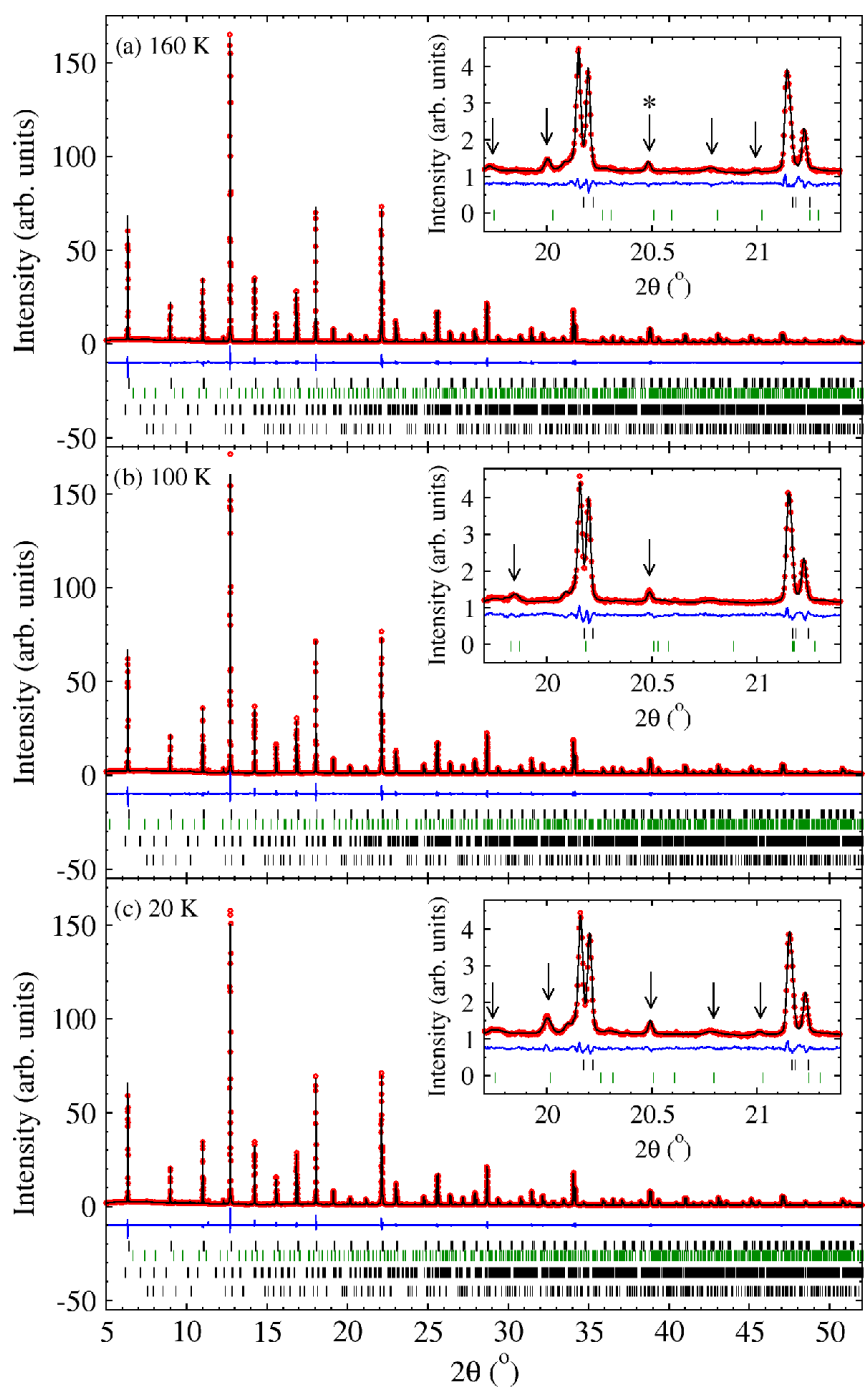

FIG. 2. Synchrotron x-ray powder diffraction data (red circles) measured at (a) $160 \mathrm{~K}$, (b) $100 \mathrm{~K}$, and (c) $20 \mathrm{~K}$. Fits of superspace structural models are shown as black lines, and the differences between $I_{\text {obs }}$ and $I_{\text {calc }}$ are given as blue lines. From top to bottom, the black tick marks show peak positions from the average $\mathrm{PbMn}_{7} \mathrm{O}_{12}$ structure, and the $\alpha-\mathrm{Mn}_{2} \mathrm{O}_{3}$ and $\mathrm{Pb}_{3}\left(\mathrm{CO}_{3}\right)_{2}(\mathrm{OH})_{2}$ impurities. The positions of incommensurate satellites originating in the structural modulation of $\mathrm{PbMn}_{7} \mathrm{O}_{12}$ are indicated by green tick marks, and highlighted in the insets by black arrows. The asterisk in the inset to (a) denotes the superimposed $(5, \overline{6}, 2)-\mathbf{k}_{\mathrm{s}}$ and $(6, \overline{5}, 2)-\mathbf{k}_{\mathrm{s}}$ peaks.

The incommensurate atomic displacements of a given site were described by modulation amplitudes $\alpha_{i}$ and $\beta_{i}$, such that the atomic fractional coordinates in a unit cell reached by the lattice vector $\left[l_{x}, l_{y}, l_{z}\right]$ are

$$
\begin{aligned}
& x=x_{0}+\alpha_{x} \sin \left(2 \pi x_{4}\right)+\beta_{x} \cos \left(2 \pi x_{4}\right)+l_{x}, \\
& y=y_{0}+\alpha_{y} \sin \left(2 \pi x_{4}\right)+\beta_{y} \cos \left(2 \pi x_{4}\right)+l_{y}, \\
& z=z_{0}+\alpha_{z} \sin \left(2 \pi x_{4}\right)+\beta_{z} \cos \left(2 \pi x_{4}\right)+l_{z},
\end{aligned}
$$

where $x_{0}, y_{0}$, and $z_{0}$ are the fractional coordinates in the average, unmodulated structure, and $x_{4}=k_{z}\left(z_{0}+l_{z}\right)$. The modulation amplitudes and the average crystal structure parameters were constrained within the $R \overline{3}(00 \gamma) 0$ superspace 
TABLE II. Modulation amplitudes (in units $\AA$ ) and refinement reliability parameters for the $R \overline{3}(00 \gamma) 0$ crystal structure of $\mathrm{PbMn}_{7} \mathrm{O}_{12}$ at 160,100 , and $20 \mathrm{~K}$. Amplitudes that are zero by symmetry are not tabulated.

\begin{tabular}{|c|c|c|c|c|}
\hline \multicolumn{2}{|c|}{$T(\mathrm{~K})$} & 160 & 100 & 20 \\
\hline \multicolumn{5}{|c|}{ Modulation amplitudes } \\
\hline $\mathrm{Pb}$ & $\alpha_{z}$ & $-0.0100(2)$ & $-0.0103(2)$ & $-0.0144(1)$ \\
\hline \multirow[t]{3}{*}{ Mn1 } & $\alpha_{x}$ & $0.0100(4)$ & $0.0119(4)$ & $0.0112(3)$ \\
\hline & $\alpha_{y}$ & $-0.0040(5)$ & $-0.0004(7)$ & $-0.0046(4)$ \\
\hline & $\alpha_{z}$ & $0.0063(7)$ & $-0.0021(6)$ & $0.0082(5)$ \\
\hline \multirow[t]{3}{*}{$\mathrm{Mn} 2$} & $\alpha_{x}$ & $0.0029(4)$ & $0.0016(5)$ & $0.0034(3)$ \\
\hline & $\alpha_{y}$ & $-0.0015(5)$ & $-0.0049(7)$ & $-0.0034(4)$ \\
\hline & $\alpha_{z}$ & $0.0030(8)$ & $-0.0012(5)$ & $0.0029(6)$ \\
\hline Mn3 & $\alpha_{z}$ & $-0.001(1)$ & $-0.002(1)$ & $-0.0056(7)$ \\
\hline \multirow[t]{6}{*}{$\mathrm{O} 1$} & $\alpha_{x}$ & $-0.006(1)$ & $-0.005(2)$ & $-0.008(1)$ \\
\hline & $\alpha_{y}$ & $0.004(1)$ & $0.001(1)$ & $0.0036(9)$ \\
\hline & $\alpha_{z}$ & $0.004(2)$ & $0.001(2)$ & $-0.001(2)$ \\
\hline & $\beta_{x}$ & $-0.004(1)$ & $-0.002(2)$ & $-0.003(1)$ \\
\hline & $\beta_{y}$ & $0.000(1)$ & $-0.003(2)$ & $0.000(1)$ \\
\hline & $\beta_{z}$ & $0.007(2)$ & $-0.003(2)$ & $0.007(2)$ \\
\hline \multirow[t]{6}{*}{$\mathrm{O} 2$} & $\alpha_{x}$ & $0.004(1)$ & $0.003(2)$ & $0.009(1)$ \\
\hline & $\alpha_{y}$ & $0.000(1)$ & $0.003(2)$ & $0.002(1)$ \\
\hline & $\alpha_{z}$ & $0.014(2)$ & $-0.010(2)$ & $0.009(2)$ \\
\hline & $\beta_{x}$ & $-0.006(1)$ & $0.001(2)$ & $-0.004(1)$ \\
\hline & $\beta_{y}$ & $0.000(1)$ & $-0.001(2)$ & $-0.001(1)$ \\
\hline & $\beta_{z}$ & $0.008(1)$ & $0.011(2)$ & $0.012(1)$ \\
\hline \multicolumn{5}{|c|}{ Reliability parameters (satellite reflections) } \\
\hline \multicolumn{2}{|c|}{$R(\%)$} & 6.8 & 5.9 & 4.8 \\
\hline \multicolumn{2}{|c|}{$w R(\%)$} & 4.9 & 4.3 & 4.2 \\
\hline
\end{tabular}

group symmetry and refined against diffraction data measured at 160, 100, and $20 \mathrm{~K}$, as shown in Fig. 2, which were chosen to represent the three incommensurate phases. The superspace group was selected based on pyrocurrent [23] and neutron powder diffraction measurements [19]. The former revealed no change in electric polarization at the transition from paramagnetic to the magnetically ordered state, indicating that the paramagnetic space group was nonpolar. The latter showed behavior consistent with coupling between the magnetic subsystem and an orbital density wave associated with the $R \overline{3}(00 \gamma) 0$ symmetry $[9,10,13]$. Excellent goodness of fit parameters were achieved, and the refined parameters and reliability factors are given in Tables I and II.

The relationship between the refined incommensurate structural modulation and the JT structural instability can be understood in terms of the Mn2-O bond lengths, which are plotted against $x_{4}$ in Figs. 3(a)-3(c). At $20 \mathrm{~K}$ [Fig. 3(a)], the $\mathrm{Mn} 2-\mathrm{O}$ bonds alternate between two long bonds along $\mathrm{x}$ and four short bonds along $\mathrm{y}$ and $\mathrm{z}$, and two long bonds along $\mathrm{y}$ and four short bonds along $\mathrm{x}$ and $\mathrm{z}$ (here $\mathrm{x}, \mathrm{y}$, and $\mathrm{z}$ define a local coordinate system with $z \sim \| c$ ). This behavior is consistent with an oscillation in the orbital occupation between $d_{3 x^{2}-r^{2}}$ and $d_{3 y^{2}-r^{2}}$, via the $d_{x^{2}-y^{2}}$ orbital, as found in $\mathrm{CaMn}_{7} \mathrm{O}_{12}$ [8]. The same qualitative behavior was observed at $160 \mathrm{~K}$ [Fig. 3(c)], albeit with a reduced amplitude. A significant change in this orbital modulation was observed at $100 \mathrm{~K}$ [Fig. 3(b)], within the quasicommensurate phase. Here

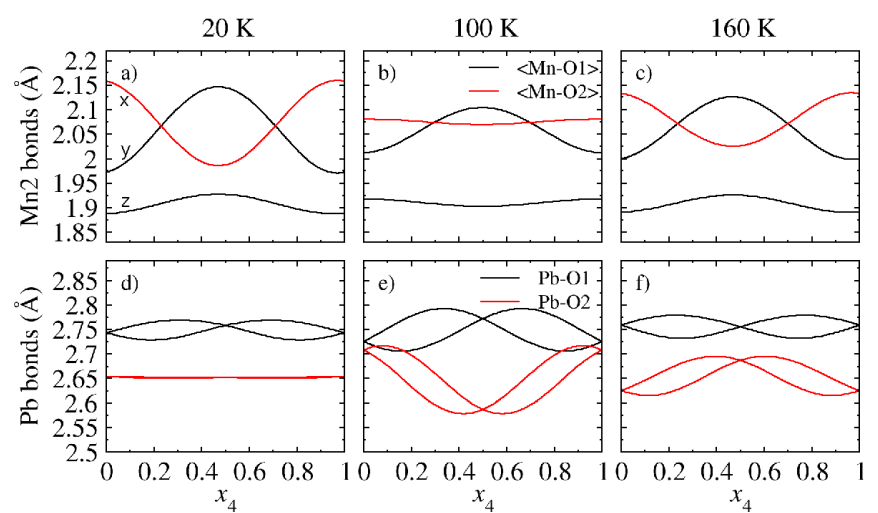

FIG. 3. (a)-(c) Averaged Mn2-O bond length pairs and (d)-(f) $\mathrm{Pb}-\mathrm{O}$ bond lengths, plotted as a function of $x_{4}$, calculated from the modulated structures refined at 20,100, and $160 \mathrm{~K}$, respectively. Six symmetry equivalent $\mathrm{Pb}-\mathrm{O} 1$ (black) and $\mathrm{Pb}-\mathrm{O} 2$ (red) bonds form the 12-fold oxygen coordination. The modulation origins are set to the $\mathrm{Mn} 2$ and $\mathrm{Pb}$ positions given in Table I. $\mathrm{x}, \mathrm{y}$, and $\mathrm{z}$ define a local coordinate system with $\mathrm{Z} \sim \| c$.

the orbital modulation associated with the JT instability had been greatly suppressed, especially along the $\mathrm{Mn} 2-\mathrm{O} 2$ bonds.

A similar analysis of $\mathrm{Pb}-\mathrm{O}$ bond lengths plotted against $x_{4}$ [Figs. 3(d) and 3(e)] elucidates the relationship between the incommensurate structural modulation and the lone electron pair structural instability. The splitting of like $\mathrm{Pb}-\mathrm{O}$ bonds, as plotted in Figs. 3(d)-3(f), is consistent with polar distortions of the $\mathrm{PbO}_{12}$ coordination. It is apparent that this splitting is largest in the quasicommensurate phase [Fig. 3(e)], moderate at $160 \mathrm{~K}$ [Fig. 3(f)], and largely suppressed at $20 \mathrm{~K}$ [Fig. 3(d)].

The local structural distortions associated with the JT and lone electron pair instabilities were quantified as follows. Based on the orbital mixing angle formalism proposed by Goodenough [24], a normalized orbital polarization $\eta\left(x_{4}\right)$ was calculated using the equation

$$
\tan \left(\frac{\pi}{3} \eta\left(x_{4}\right)\right)=\sqrt{3} \frac{\overline{\mathrm{x}}\left(x_{4}\right)-\overline{\mathrm{y}}\left(x_{4}\right)}{\overline{\mathrm{x}}\left(x_{4}\right)+\overline{\mathrm{y}}\left(x_{4}\right)-2 \overline{\mathrm{z}}\left(x_{4}\right)},
$$

where $\overline{\mathbf{X}}\left(x_{4}\right), \overline{\mathbf{y}}\left(x_{4}\right)$, and $\overline{\mathbf{z}}\left(x_{4}\right)$ are the average lengths of opposite $\mathrm{Mn}-\mathrm{O}$ bond pairs. By this definition $\eta=0$ corresponds to occupation of the $d_{x^{2}-y^{2}}$ orbital, and $\eta= \pm 1$ correspond to maximal occupation of the $d_{3 x^{2}-r^{2}}$ and $d_{3 y^{2}-r^{2}}$ orbitals, respectively. The $\mathrm{Pb}$ polarization $\zeta\left(x_{4}\right)$ was taken to be the difference between the center of mass of the $12 \mathrm{~Pb}$ oxygen ligands at positions $\mathbf{r}_{\mathrm{O}_{i}}\left(x_{4}\right)$ and the central $\mathrm{Pb}$ position $\mathbf{r}_{\mathrm{Pb}}\left(x_{4}\right)$ :

$$
\zeta\left(x_{4}\right)=\left|\frac{1}{12} \sum_{i=1}^{12} \mathbf{r}_{\mathrm{O}_{i}}\left(x_{4}\right)-\mathbf{r}_{\mathrm{Pb}}\left(x_{4}\right)\right|,
$$

where $\mathbf{r}_{\mathrm{O}_{i}}\left(x_{4}\right)$ and $\mathbf{r}_{\mathrm{Pb}}\left(x_{4}\right)$ were defined in a Cartesian basis in units $\AA$.

Figure 4(a) shows the maximum absolute value of the $d_{3 x^{2}-r^{2}}$ and $d_{3 y^{2}-r^{2}}$ normalized orbital polarizations evaluated on cooling from 300 down to $5 \mathrm{~K}$. The orbital polarization was found to grow below $T_{\mathrm{OO} 1}$, and reached a maximum value of 0.6 (60\% polarized) at the lowest measured temperature. This value is considerably reduced compared to the $95 \%$ orbital polarization observed in $\mathrm{CaMn}_{7} \mathrm{O}_{12}$ [8], indicating partial 


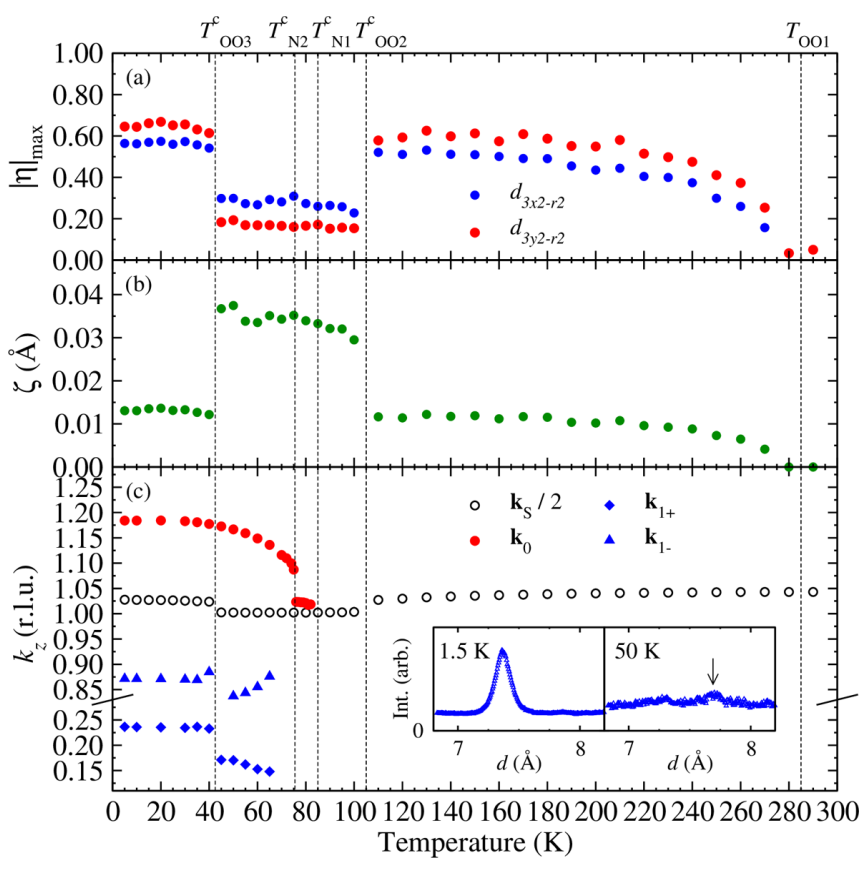

FIG. 4. Temperature dependence of (a) maximum values of the $d_{3 x^{2}-r^{2}}$ and $d_{3 y^{2}-r^{2}}$ normalized orbital polarization, (b) the $\mathrm{Pb}$ polarization averaged over $x_{4}$, and (c) the magnetic propagation vectors reproduced from Ref. [19] plotted alongside $\mathbf{k}_{\mathrm{s}} / 2$. All values were determined from data measured on cooling. The inset to (c) highlights $\mathbf{k}_{1-}$ magnetic diffraction peaks measured at 1.5 (left) and $50 \mathrm{~K}$ (right, marked by a black arrow), also reproduced from Ref. [19].

suppression of the orbital order at all temperatures. The quasicommensurate phase between $T_{\mathrm{OO} 3}$ and $T_{\mathrm{OO} 2}$ is characterized by a large drop in both orbital polarizations, consistent with the change in bond lengths plotted in Fig. 3. The opposite behavior was observed in the temperature dependence of the $\mathrm{Pb}$ polarization, which is shown averaged over $x_{4}$ in Fig. 4(b). A small $\mathrm{Pb}$ polarization evolved below $T_{\mathrm{OO} 1}$, which became greatly enhanced in the quasicommensurate phase.

These results demonstrate that the new quasicommensurate structural phase of $\mathrm{PbMn}_{7} \mathrm{O}_{12}$ arises due to competition between Mn2 JT and Pb lone-pair instabilities. The nature of this competition and the implications for long-range magnetic order are discussed in the following section.

\section{DISCUSSION}

The role of $\mathrm{Pb}$ lone-pair instabilities in establishing the quasicommensurate phase can be understood by considering the crystal structure in the commensurate limit $\mathbf{k}_{s}=(0,0,2)$. The symmetry of this structure depends on the global phase of the modulation, and as such can be either centrosymmetric $P \overline{3}$ or noncentrosymmetric $P 3$. In the former case, only sine terms are present $\left(\beta_{i}=0\right)$, while in the latter, an admixture of sine and cosine terms is allowed. Let us consider the more symmetric structure $P \overline{3}$. In this structure, two out of the three $\mathrm{Pb}$ sites related by $R$-centering translation of the parent structure obtain a large polarization, while one site has zero polarization. The $\mathrm{Pb}$ polarization is stabilized by the formation of asymmetric short $\mathrm{Pb}-\mathrm{O}$ bonds. In the layer of atoms centered around $z=1 / 2$, short $\mathrm{Pb}-\mathrm{O}$ bonds and (a)

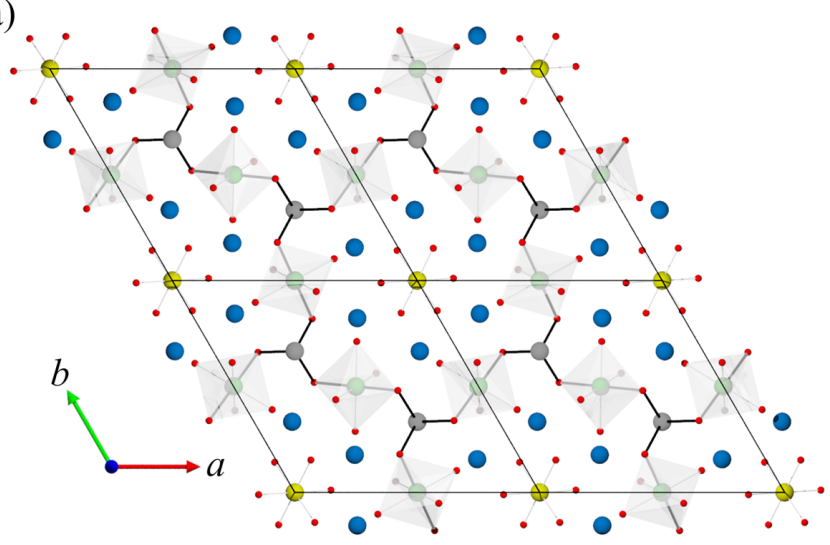

(b)

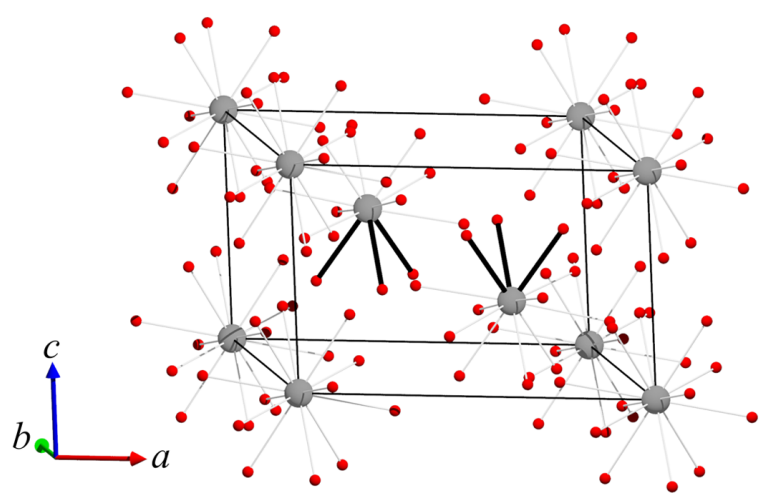

FIG. 5. The quasicommensurate crystal structure of $\mathrm{PbMn}_{7} \mathrm{O}_{12}$ drawn in the commensurate limit, in which the $\mathrm{Pb}$ lone-pair instability is maximally stabilized (see text). (a) The layer of atoms centered around $z=1 / 2$, (b) $3 \mathrm{D}$ view of $\mathrm{PbO}_{12}$ coordinations. $\mathrm{Pb}, \mathrm{Mn} 1, \mathrm{Mn} 2$, $\mathrm{Mn} 3$, and $\mathrm{O}$ atoms are drawn as gray, blue, green, yellow, and red spheres, respectively. JT active Mn2 octahedra are shaded gray. Short $\mathrm{Pb}-\mathrm{O}$ bonds are highlighted in bold, and the commensurate unit cell is drawn as fine black lines.

Mn2 JT elongation axes form a coherent honeycomb motif [Fig. 5(a)] in which both structural instabilities are satisfied. However, in the layer of atoms centered around $z=0$ (not shown), both $\mathrm{Pb}$ lone-pair and $\mathrm{Mn} 2$ orbital polarizations are zero. Figure 5(b) gives a three-dimensional (3D) view of the commensurate unit cell that highlights the antiferroelectric arrangement of polar $\mathrm{PbO}_{12}$ distortions at the two $\mathrm{Pb}$ sites in the center of the commensurate unit cell. Assuming that in the layers with the antiferroelectric distortions the lone-pair instability is satisfied, the absolute value of the local polarization, averaged over the period of the commensurate modulation, is $2 / 3 \approx 0.667$ of the hypothetical "fully polarized state." In the case of an incommensurate modulation, the averaged polarization is $2 / \pi \approx 0.637$. Thus, the commensurate antiferroelectric structure better optimizes the lone-pair electronic instability.

Despite the favorable relationship between the lone-pair instability and the commensurate structure, we find that the refined propagation vector is slightly off the commensurate value in the quasicommensurate phase. This experimental finding might be associated with the formation of an ordered pattern of commensurate translational domains with modulation (phase) slips at the domain boundaries. By this 
mechanism the system can systematically omit structural layers with zero polarization and thereby increase the density of the layers shown in Fig. 5(a). In this case the frequent repetition of the translational domains result in a discrete (steplike) modulated state that gives incommensurate satellite reflections in diffraction experiments. This is a well-known phenomenon for some alloys with so-called long period antiphase boundary modulated structures [25]. Furthermore, an anharmonic steplike incommensurate structural modulation was proposed for another perovskite system with a lone-pair electronic instability: $\mathrm{Bi}_{0.75} \mathrm{La}_{0.25} \mathrm{FeO}_{3}$ [26]. To some extent, this model can also be considered as an ordered pattern of antiphase domains of a commensurate antipolar structure.

$\mathrm{PbMn}_{7} \mathrm{O}_{12}$ was reported to undergo three, low temperature magnetic phase transitions at $T_{\mathrm{N} 1}=83 \mathrm{~K}, T_{\mathrm{N} 2}=77 \mathrm{~K}$, and $T_{\mathrm{N} 3}=43 \mathrm{~K}$, with a large thermal hysteresis observed at $T_{\mathrm{N} 3}$ between approximately 37 and $65 \mathrm{~K}$ [23]. In the first magnetically ordered phase $\left(T_{\mathrm{N} 2}<T<T_{\mathrm{N} 1}\right), \mathrm{PbMn}_{7} \mathrm{O}_{12}$ was found to adopt the same lock-in magnetic structure as $\mathrm{CaMn}_{7} \mathrm{O}_{12}$ [19], albeit over a much narrower temperature range. The ground state magnetic structure of $\mathrm{PbMn}_{7} \mathrm{O}_{12}\left(T<T_{\mathrm{N} 3}\right)$ was found to be a delocked helix with modulated spin chirality (also observed in the ground state of $\mathrm{CaMn}_{7} \mathrm{O}_{12}[13,19]$ ); evidenced by $\mathbf{k}_{1+}$ and $\mathbf{k}_{1-}$ magnetic diffraction peaks. According to the phenomenological model of magneto-orbital coupling [13], the observation of a modulated spin chirality below $T_{\mathrm{N} 3}$ implied the presence of an incommensurate orbital density wave with $R \overline{3}(00 \gamma) 0$ symmetry. This inference is now confirmed by the results discussed in Sec. III.

Remarkably, in the intermediate magnetic phase of $\mathrm{PbMn}_{7} \mathrm{O}_{12}\left(T_{N 3}<T<T_{N 2}\right)$ the fundamental magnetic order was found to be delocked as in the ground state, but the $\mathbf{k}_{1+}$ and $\mathbf{k}_{1-}$ magnetic diffraction peaks associated with the magneto-orbital coupling were apparently missing. This result indicated that either the magnetic and orbital orders unexpectantly decoupled, or that the orbital modulation was temporarily destroyed [19]. The above results demonstrate that the magnetic phase transition at $T_{N 3}$ is in fact a magnetostructural phase transition with $T_{\mathrm{N} 3}=T_{\mathrm{OO} 3}$. This relationship is exemplified by the large thermal hysteresis observed at both $T_{\mathrm{N} 3}$ and $T_{\mathrm{OO} 3}$. Hence, the intermediate magnetic phase occurs due to the stabilization of the concomitant quasicommensurate structural phase.

The phenomenological relationship between magnetic and structural propagation vectors that holds in the ground state implies that the $\mathbf{k}_{1+}$ and $\mathbf{k}_{1-}$ magnetic peaks in the intermediate phase will have shifted position in accordance with the newly established quasicommensurate propagation vector. Furthermore, the large suppression of the orbital order in the quasicommensurate phase would lead to a suppression in the spin-chirality modulation and hence a reduction in the $\mathbf{k}_{1+}$ and $\mathbf{k}_{1-}$ diffraction intensities. Careful inspection of the neutron powder diffraction data reported in Ref. [19] [reproduced in the Fig. 4(c) inset] showed the presence of weak diffrac- tion peaks at the shifted $\mathbf{k}_{1-}$ position, close to the detection limit of the experiment. The temperature dependence of the measured $\mathbf{k}_{1-}$ and calculated $\mathbf{k}_{1+}$ (based upon the former) propagation vectors within the intermediate phase is shown in Fig. 4(c), alongside the other magnetic propagation vectors also reproduced from Ref. [19]. Thus, in light of the quasicommensurate structural phase, the unusual magnetic behavior of $\mathrm{PbMn}_{7} \mathrm{O}_{12}$ can be naturally understood based on the above structural characterization and the phenomenological model of the magneto-orbital coupling, previously developed to explain the lock-in phase and multi-k magnetic ground state in $\mathrm{CaMn}_{7} \mathrm{O}_{12}$ [13].

\section{CONCLUSIONS}

The quadruple perovskite manganite $\mathrm{PbMn}_{7} \mathrm{O}_{12}$ exhibits a set of structural phase transitions upon cooling below room temperature. The higher temperature transition at $T_{\mathrm{OO} 1}=$ $294 \mathrm{~K}$ is associated with development of an orbital density wave with incommensurate propagation vector $\mathbf{k}_{s}=$ $(0,0, \sim 2.08)$. At $T_{\mathrm{OO} 2}=110 \mathrm{~K}$, the propagation vector suddenly changes taking the nearly commensurate value $\mathbf{k}_{s}=$ $(0,0,2.0060(6))$. The quasicommensurate modulation holds down to $T_{\mathrm{OO} 3}=40 \mathrm{~K}$, when a re-entrant transition back to the incommensurate phase takes place. The orbital density wave is significantly suppressed in the quasicommensurate phase while the structural distortions optimizing the lone-pair electronic instability of $\mathrm{Pb}^{2+}$ are enhanced. This behavior is interpreted as a competition between the lone-pair and JT instabilities. The magnetic order that emerges in the quasicommensurate phase reflects these structural changes specific to this perovskite. In particular, the puzzling absence of the magneto-orbital coupling and associated modulation of the spin chirality reported in the intermediate magnetic phase $(40<T<65 \mathrm{~K})$, is due to the severe reduction of the orbital density wave in the quasicommensurate regime. The re-entrant transition to the incommensurate structural phase re-establishes the orbital polarization which in turn triggers a strong magneto-orbital coupling in the ground state.

\section{ACKNOWLEDGMENTS}

R.D.J. acknowledges support from a Royal Society University Research Fellowship. A.A.B. acknowledges partial support from JSPS KAKENHI (Grant No. JP20H05276), a research grant (40-37) from Nippon Sheet Glass Foundation for Materials Science and Engineering, and Innovative Science and Technology Initiative for Security (Grant No. JPJ004596) from Acquisition, Technology, and Logistics Agency (ATLA), Japan. We acknowledge SOLEIL for provision of synchrotron radiation facilities (Proposal No. 20171110) and we would like to thank E. Elkaim for assistance in using beamline Cristal.
[1] K. I. Kugel and D. I. Khomskii, Sov. Phys. Usp. 25, 231 (1982).

[2] J. B. Goodenough, Annu. Rev. Mater. Sci. 28, 1 (1998).
[3] J. B. Goodenough, Phys. Rev. 100, 564 (1955).

[4] E. O. Wollan and W. C. Koehler, Phys. Rev. 100, 545 (1955). 
[5] P. G. Radaelli, D. E. Cox, M. Marezio, and S.-W. Cheong, Phys. Rev. B 55, 3015 (1997).

[6] B. Bochu, J. L. Buevoz, J. Chenavas, A. Collomb, J. C. Joubert, and M. Marezio, Solid State Comm. 36, 133 (1980).

[7] D. Khomskii and J. van den Brink, Phys. Rev. Lett. 85, 3329 (2000).

[8] N. Perks, R. Johnson, C. Martin, L. Chapon, and P. Radaelli, Nat. Commun. 3, 1277 (2012).

[9] W. Sławiński, R. Przeniosło, I. Sosnowska, M. Bieringer, I. Margiolaki, and E. Suard, Acta Crystallogr. Sect. B 65, 535 (2009).

[10] W. Sławiński, R. Przeniosło, I. Sosnowska, and M. Bieringer, J. Phys.: Condens. Matter 22, 186001 (2010).

[11] R. D. Johnson, L. C. Chapon, D. D. Khalyavin, P. Manuel, P. G. Radaelli, and C. Martin, Phys. Rev. Lett. 108, 067201 (2012).

[12] W. Sławiński, R. Przeniosło, I. Sosnowska, and V. Petříček, Acta Crystallogr. Sect. B 68, 240 (2012).

[13] R. D. Johnson, D. D. Khalyavin, P. Manuel, A. Bombardi, C. Martin, L. C. Chapon, and P. G. Radaelli, Phys. Rev. B 93, 180403(R) (2016).

[14] F. Mezzadri, G. Calestani, M. Calicchio, E. Gilioli, F. Bolzoni, R. Cabassi, M. Marezio, and A. Migliori, Phys. Rev. B 79, 100106(R) (2009).

[15] A. A. Belik, Y. Matsushita, Y. Kumagai, Y. Katsuya, M. Tanaka, S. Y. Stefanovich, B. I. Lazoryak, F. Oba, and K. Yamaura, Inorg. Chem. 56, 12272 (2017).
[16] J. Rodríguez-Carvajal, M. Hennion, F. Moussa, A. H. Moudden, L. Pinsard, and A. Revcolevschi, Phys. Rev. B 57, R3189(R) (1998).

[17] W. A. Sławiński, H. Okamoto, and H. Fjellvåg, Acta Crystallogr. Sect. B 73, 313 (2017).

[18] D. D. Khalyavin, R. D. Johnson, F. Orlandi, P. G. Radaelli, P. Manuel, and A. A. Belik, Science 369, 680 (2020).

[19] R. D. Johnson, D. D. Khalyavin, P. Manuel, P. G. Radaelli, I. S. Glazkova, N. Terada, and A. A. Belik, Phys. Rev. B 96, 054448 (2017).

[20] V. Petříček, M. Duček, and L. Palatinus, Z. Kristallogr. Cryst. Mater. 229, 345 (2014).

[21] T. Locherer, R. Dinnebier, R. Kremer, M. Greenblatt, and M. Jansen, J. Solid State Chem. 190, 277 (2012).

[22] A. A. Belik, Y. S. Glazkova, Y. Katsuya, M. Tanaka, A. V. Sobolev, and I. A. Presniakov, J. Phys. Chem. C 120, 8278 (2016).

[23] A. A. Belik, Y. S. Glazkova, N. Terada, Y. Matsushita, A. V. Sobolev, I. A. Presniakov, N. Tsujii, S. Nimori, K. Takehana, and Y. Imanaka, Inorg. Chem. 55, 6169 (2016).

[24] J. B. Goodenough, Magnetism and the Chemical Bond (John Wiley and Sons, New York, 1963).

[25] J. Van Landuyt, G. Van Tendeloo, and S. Ainellnckx, Pure Appl. Chem. 57, 1373 (1985).

[26] D. A. Rusakov, A. M. Abakumov, K. Yamaura, A. A. Belik, G. Van Tendeloo, and E. Takayama-Muromachi, Chem. Mater. 23, 285 (2011). 